\title{
A knowledge coverage-based trust propagation for recommendation mechanism in social network group decision making
}

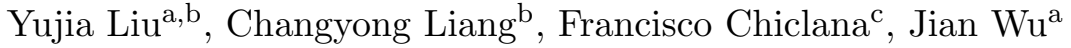 \\ ${ }^{a}$ School of Economics and Management, Shanghai Maritime University, Shanghai201306, China \\ ${ }^{b}$ School of Management, Hefei University of Technology, Hefei, Anhui, China \\ ${ }^{c}$ Institute of Artificial Intelligence, Faculty of Technology, De Montfort University, Leicester, UK
}

\begin{abstract}
Trust is a typical relationship in social network, which in group decision making problems relates to the inner relationship among experts. To obtain a complete trust relationship of a networked group of experts, firstly, a novel knowledge coverage-based trust propagation operator is proposed to estimate the trust relationship between pairs of unknown experts. The novelty of this trust propagation operator resides in its account of the domain knowledge coverage of experts. Desirable properties regarding boundary conditions, generalisation and knowledge coverage absorption are studied. The comparison with existing operators of boundary conditions shows the rationality of the proposed operator. Next, a knowledge coverage-based multi-paths trust propagation model for constructing complete trust network is investigated. The proposed approach aggregates all trust paths to collect all trust information and penalise trust decay. Secondly, a trust order induced recommendation mechanism is proposed by combining subjective and objective weights. Thus, experts can accept consensus recommendations by subjective and objective trust. This recommendation mechanism allows the inconsistent experts to accept the advices they trust. The validity and rationality of the proposed recommendation mechanism is mathematically proved, and a numerical example is utilised to illustrate the calculation process of the proposed method.
\end{abstract}

Keywords: Group decision making, Social network analysis, Recommendation mechanism, Trust propagation, Knowledge coverage

\section{Introduction}

Since experts in decision panels come from different areas, they may have different knowledge backgrounds, which will lead to conflict and inconsistency of group opinions [1-3]. The independent moderator usually requires a certain minimum consensus to ensure the rationality of decision made by a panel [4]. Therefore, how to reach such a minimum group consensus is a key research topic in multiple criteria group decision-making (MCGDM).

Email addresses: lyj71@126.com (Yujia Liu), cyliang@hfut.edu.cn (Changyong Liang), chiclana@dmu.ac.uk (Francisco Chiclana), jyajian@163.com (Jian Wu) 
Interactive mechanisms for group consensus have been proposed to make the decision process more intelligent by automatically triggering inconsistent decision opinions detection and recommendation mechanisms [5-8]. However, the common idea of these interactive mechanisms relies in the forced acceptance by the inconsistent experts of the recommendations based on the average preference of the other experts in the group [9], which in practice has been argued to be an unreasonable approach because it neglects completely the trust relationships between experts, which can provide a practical and rational support for group consensus $[10,11]$.

Social networks, such as Twitter (http://www.twitter.com/), show that users accept advices that come from individuals they trust during [12]. In group decision situations, the inconsistent experts tend to accept the advice based on the opinions of other experts they trust [13, 14]. Liu et al. [15] proposed a recommendation mechanism for reaching group consensus that involved measuring the trust degree between pairs of experts. In contrast to the interactive mechanisms referred to above, trust induced recommendation mechanisms enhance the self-esteem of the inconsistency experts because they are provided advice from the experts they trust, which can be unforcedly accepted. However, the existing trust relationship based recommendations mechanisms refer to objective trust only, i.e. a trust measure based on the similarity degree of decision opinions. Since subjective trust is an essential part of trust relationships, it should also play an important role in the recommendation mechanism. Thus, a recommendation mechanism induced by both objective and subjective trust will be a more rational approach to reaching group consensus. This is the aim of this this study, and a trust order induced consensus reaching mechanism is proposed, where the induced weight vector is obtained via the aggregation of both subjective weight vector, obtained from the trust network of the group of experts, and objective weight vector, obtained from the consensus degree of experts' decision matrices.

Trust propagation is a practical method for solving the data sparsity of trust networks [16]. Indeed, some experts may not have a direct trust relationship with other experts in the network but they may have several indirect trust relationship with such experts via trusted third party (TTP) [17-19]. The assumption of trust propagating through such indirect paths has been used to build approaches to estimate the unknown direct trust relationship between experts and, subsequently, to obtain a complete trust network $[1,20]$. Trust relationship is a kind of expert's subjective opinion. On the other hand, the decision result is very likely to rely on the domain knowledge degree of experts [21]. The effect of knowledge degree on group decision results has been increasingly acknowledged by researchers [22, 23], with some researchers noticing that knowledge degree even affects the confidence of decision-makers [24-26]. The trust relation with low knowledge in the trust propagation process may lead to a debatable evaluation of trust degree for unknown experts, and decrease the accuracy of decision results. It is reasonable to claim that a knowledge degree is key in constructing a trust network and the decisionmaking problem based on it. However, existing studies of trust propagation operators do not consider 
the knowledge degree. In real life, people trust others with a degree which is not a 0-1 variable of total 'trust or distrust', but with reacher ranges that could lead to the following four concepts: trust, distrust, hesitancy and conflict [27]. To account for knowledge degree, this study proposes a concept of knowledge coverage (KC) to quantify the domain knowledge degree of experts. Based on the $\mathrm{KC}$ concept, this study proposes a knowledge coverage-based trust propagation operator. Then, for avoiding losing useful trust information [28, 29], a knowledge coverage-based multi-paths trust propagation model is developed on the basis of a $\mathrm{KC}$ based trust propagation operator to aggregate trust information of multiple trust paths that, at the same time, penalises the longer paths that lead to higher trust decay.

This rest of the paper is set out as follows: Section 2 introduces the required concepts and modelling associated to an incomplete trust social network. The basic expression of a trust relationship is introduced; definitions of trust score space, trust functions, and its associated knowledge deficit are also presented. It also recall the existent t-norm and uninorm based trust propagation operators. Section 3 introduces the novel concept of knowledge coverage, proposes a ranking method of trust functions, and elaborate on the development of a knowledge coverage-based trust propagation operator is developed. Subsequently, its desirable propositions are investigated. A knowledge coverage-based multi-paths trust propagation model is proposed to estimate unknown trust relationships and to obtain a complete trust network. Section 3 also proposes a novel trust order induced consensus reaching mechanism to reach the group consensus, which is driven by an induced weight vector derived from both subjective trust degrees and objective trust degrees. Section 4 focuses on the selection process to obtain the final consensus based decision. Conclusions are drawn in section 5. Besides, along the paper a numerical example of cloud services selection is used to illustrate the proposed method computation process.

\section{Preliminaries}

\subsection{Trust social network}

Social network analysis (SNA) is a widely used method that focuses on the relationship between members in social networks. Trust is a representative relationship in social networks. There are three general representations of SNA:

- Graph - social networks can be represented by nodes and edges with directions (Fig.1).

- Algebraic - social network can be described by listing the relationship between pairs of members in the social networks. The graphic representation of the social network in Fig.1 can be algebraically represented as:

$E_{1} R E_{2} \quad E_{1} R E_{3} \quad E_{1} R E_{4} \quad E_{2} R E_{4} \quad E_{2} R E_{5} \quad E_{3} R E_{2} \quad E_{4} R E_{3} \quad E_{4} R E_{5} \quad E_{5} R E_{1} \quad E_{5} R E_{3}$ 


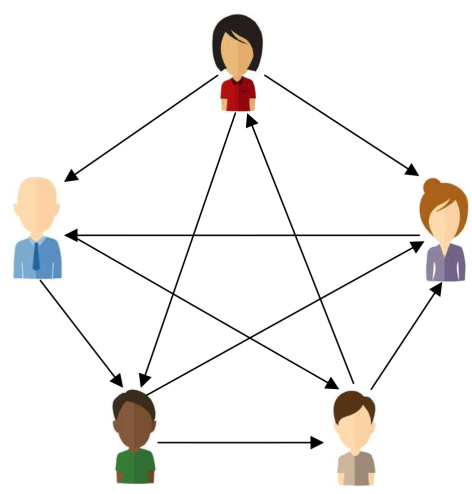

Figure 1: Graphic representation of a social network

- Sociometric: use "1" and "0" to represent "have" and "don't have" for a two-way relationship, respectively. The relationship in Fig.1 can be represent as:

$$
\left[\begin{array}{lllll}
0 & 1 & 1 & 1 & 0 \\
0 & 0 & 0 & 1 & 1 \\
0 & 1 & 0 & 0 & 0 \\
0 & 0 & 1 & 0 & 1 \\
1 & 0 & 1 & 0 & 0
\end{array}\right]
$$

The above representations of a social network show only the existence (1) or lack of (0) relationship between any two nodes. In practical situations, social members' relationship strengths are not all equally weighted. In particular, when the strength of relationship is related to the concept of 'trust', the network is referred to a trust network [30].

\subsection{Trust score space and ranking method}

Definition 1 (Trust score space). A trust score space can be defined as:

$T S S=\left([0,1]^{2}, \leq_{t d}, \leq_{k}, \neg\right)$

where $[0,1]^{2}$ is a bilattice trust score; $\leq_{t d}$ is the trust-distrust ordering; $\leq_{k}$ is the knowledge ordering; and $\neg$ is a negation operator, satisfying the following properties:

- $\left(t_{1}, d_{1}\right) \leq_{t d}\left(t_{2}, d_{2}\right)$ iff $t_{1} \leq t_{2}$ and $d_{1} \geq d_{2}$

- $\left(t_{1}, d_{1}\right) \leq_{k}\left(t_{2}, d_{2}\right)$ iff $t_{1} \leq t_{2}$ and $d_{1} \leq d_{2}$

- $\neg\left(t_{1}, d_{1}\right)=\left(d_{1}, t_{1}\right)$ for all $\left(t_{1}, d_{1}\right)$ and $\left(t_{2}, d_{2}\right)$ in $[0,1]^{2}$

Definition 2 (Trust function). An element of a trust score space, $(t, d) \in[0,1]^{2}$, with $t$ representing a trust degree, and $d$ a distrust degree, is called a trust function and it is denoted as $T F=(t, d)$. 


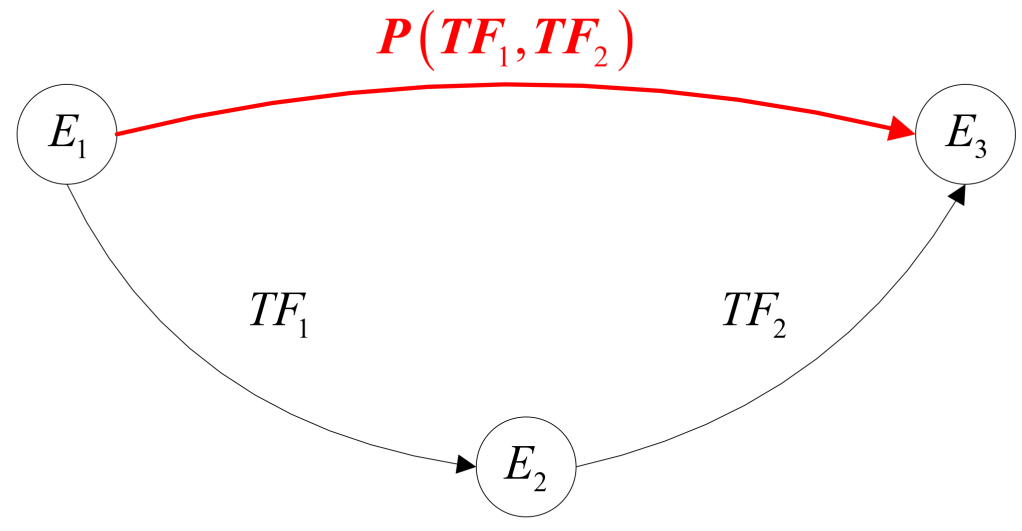

Figure 2: Trust propagation

Definition 3 (Knowledge deficit). The knowledge deficit of a trust function $(t, d) \in[0,1]^{2}$ is

$k d=(1-t-d)^{2}$

Based on the concept of knowledge deficit, the following types of information are discriminated:

a) Incomplete knowledge iff $t+d<1$.

b) Inconsistent knowledge iff $t+d>1$.

c) Perfect knowledge iff $t+d=1$.

Fig.1 represents an incomplete network, i.e. a network lacking trust relationships between some of its nodes. Thus, for such pairs of nodes, their trust values are unknown, which is a universal phenomenon [31] as most networks are incomplete. Therefore, estimating the trust relationship from an individual/organisation to an unknown individual/organisation is a significant question in SNA. Existing studies have developed trust propagation methods for unlinked individuals/organisations via trusted third party (TTP) having direct trust to each other as as Fig.2 shows [32, 33]. Victor et al. [30] argued that the opinions of trusted people are easier to accept, while opinions from a distrusted or unknown third party could be ignored, which was used to developed a trust propagation operator based on t-norms.

Definition 4 (T-norm propagation operator). A t-norm, $\mathcal{T}$, based propagation operator of trust functions $T F_{1}=\left(t_{1}, d_{1}\right)$ and $T F_{2}=\left(t_{2}, d_{2}\right)$ is

$\mathcal{P}_{v}\left(\left(t_{1}, d_{1}\right),\left(t_{2}, d_{2}\right)\right)=\left(\mathcal{T}\left(t_{1}, t_{2}\right), \mathcal{T}\left(t_{1}, d_{2}\right)\right)$

Wu et al. [20] developed a trust propagation operator based on uninorm that addressed drawbacks associated to Victor's trust propagation operators assumption of trust and distrust being considered independent. 
Definition 5 (Uninorm trust propagation operator). A uninorm, $U$, based propagation operator of trust functions $T F_{1}=\left(t_{1}, d_{1}\right)$ and $T F_{2}=\left(t_{2}, d_{2}\right)$ is

$\mathcal{P}_{U}\left(\left(t_{1}, d_{1}\right),\left(t_{2}, d_{2}\right)\right)=\left(U\left(t_{1}, t_{2}\right), U\left(t_{1}, d_{2}\right)\right)$

\section{Knowledge coverage-based multi-paths trust propagation method}

The above two types of trust propagation operators, which are valid propagating trust, ignore concept of knowledge coverage in trust relationships. In addition, both propagation operators are based on the shortest path rule, which means that they ignore the trust information in the rest trust paths that are not utilised. In addition, the trust decay is not accounted for [34, 35]. This study investigates a knowledge coverage-based multi-paths trust propagation model on the basis of a knowledge coverage based trust propagation operator to aggregate trust information of multiple trust paths that, at the same time, penalises the longer paths that lead to higher trust decay.

Knowledge deficit can be viewed as a negative degree. Knowledge coverage, as the negation of knowledge deficit, is therefore a positive degree.

Definition 6 (Knowledge coverage). Given a trust function $(t, d) \in[0,1]^{2}$, the value

$k c=1-(1-t-d)^{2}$

Is known as its knowledge coverage.

A trust function with $k c=1(\Longleftrightarrow t+d=1)$ has a complete knowledge coverage; while a trust function with $k c=0(\Longleftrightarrow t+d=0 \vee t+d=2 \Longleftrightarrow(t, d) \in\{(0,0),(1,10\})$ has no knowledge coverage. Inspired by the correlation coefficient proposed by Gerstenkorn and Manko [36], this study proposes a definition of positive trust score, which calculates the correlation coefficient between a trust function and perfect trust function $\left(T F^{*}=(1,0)\right)$ to rank trust functions.

Definition 7 (Positive trust score). The positive trust score (PTS) of a trust functions is

$\operatorname{PTS}(t, d)= \begin{cases}\frac{t}{\sqrt{t^{2}+d^{2}}} & , \text { if }(t, d) \neq(0,0) \\ 0 & , \text { if }(t, d)=(0,0)\end{cases}$

Definition 8 (Ranking method). Let $T F_{1}=\left(t_{1}, d_{1}\right)$ and $T F_{2}=\left(t_{2}, d_{2}\right)$ be two trust functions; $P T S_{1}$ and $P T S_{2}$ their positive trust score; and $k c_{1}$ and $k c_{2}$ their knowledge coverage, respectively.

$$
T F_{1}<T F_{2} \Longleftrightarrow\left[P T S_{1}<P T S_{2}\right] \vee\left[P T S_{1}=P T S_{2} \wedge k c_{1}<k c_{2}\right] .
$$

where $\vee$ is the logical OR; $\wedge$ is the logical AND.

The above ranking method makes the comparison of decision opinions expressed by trust functions feasible. The positive trust score and knowledge coverage degree are used to establish the ordering $T F s$, which will be exploited later at the advice generation within the consensus interaction mechanism. 


\subsection{Knowledge coverage-based trust propagation operator}

The above t-norm and uninorm based trust propagation operators treat trust values with different knowledge deficit equally. This study proposes a knowledge coverage-based trust propagation operator that retains the conflicting information while, at the same time, implementing the knowledge coverage of experts.

Notice that, in practical situations, if expert $E_{1}$ fully distrust expert $E_{2}\left(T F_{12}=(0,1)\right)$ while, at the same time, the knowledge coverage of expert $E_{2}$ on expert $E_{3}$ is maximum/complete, i.e., $k c_{23}=1$, then the propagation result of how much $E_{1}$ trust $E_{2}$ would result in $T F_{13}=(0,1)$. The same result would be expected when expert $E_{2}$ fully distrust expert $E_{3}$ while, at the same time, the knowledge coverage of expert $E_{1}$ on expert $E_{2}$ is the complete. Thus, the knowledge coverage-based trust propagation operator proposed in this study is defined below:

Definition 9 (Knowledge coverage-based trust propagation operator). Let $T F_{1}=\left(t_{1}, d_{1}\right)$ and $T F_{2}=\left(t_{2}, d_{2}\right)$ be two trust functions, and and $k c_{1}$ and $k c_{2}$ their knowledge coverage, respectively. The knowledge coverage-based trust propagation operator is

$$
\begin{aligned}
& \mathcal{P}_{k c}\left(T F_{1}, T F_{2}\right)=\mathcal{P}_{k c}\left(\left(t_{1}, d_{1}\right),\left(t_{2}, d_{2}\right)\right) \\
& = \begin{cases}(0,1) & , \text { if } T F_{1}=(0,1) \wedge k c_{2}=1 \\
(0,1) & , \text { if } T F_{2}=(0,1) \wedge k c_{1}=1 \\
\left(U\left(\mathcal{T}\left(k c_{1}, t_{1}\right), \mathcal{T}\left(k c_{2}, t_{2}\right)\right), U\left(\mathcal{T}\left(k c_{1}, t_{1}\right), \mathcal{T}\left(k c_{2}, d_{2}\right)\right)\right) & , \text { otherwise. }\end{cases}
\end{aligned}
$$

where $U$ is a uninorm and $\mathcal{T}$ is a t-norm.

For the case of being $U$ the cross ratio uninorm and $\mathcal{T}$ the product t-norm, the expression of the knowledge coverage-based trust propagation operator is:

$$
\begin{aligned}
& \mathcal{P}_{k c}\left(T F_{1}, T F_{2}\right)=\mathcal{P}_{k c}\left(\left(t_{1}, d_{1}\right),\left(t_{2}, d_{2}\right)\right) \\
& = \begin{cases}(0,1) & , \text { if } T F_{1}=(0,1) \wedge k c_{2}=1 \\
(0,1) & , \text { if } T F_{2}=(0,1) \wedge k c_{1}=1 \\
\left(\frac{t_{1} k c_{1} t_{2} k c_{2}}{t_{1} k c_{1} t_{2} k c_{2}+\left(1-t_{1} k c_{1}\right)\left(1-t_{2} k c_{2}\right)}, \frac{t_{1} k c_{1} d_{2} k c_{2}}{t_{1} k c_{1} d_{2} k c_{2}+\left(1-t_{1} k c_{1}\right)\left(1-d_{2} k c_{2}\right)}\right) & , \text { otherwise. }\end{cases}
\end{aligned}
$$

The knowledge coverage-based trust propagation operator is non-commutative and non-associative. However, the knowledge coverage-based trust propagation operator has the following properties.

Property 1 (Boundary values). The values of the knowledge coverage-based trust propagation operator with trust function inputs in the set $\{(0,0),(0,1),(1,0),(1,1)\}$ are in Table 1 . In other words, the knowledge coverage-based trust propagation operator verifies the symmetry (commutativity) property in the extreme boundary points of the domain $[0,1]^{2}$.

Property 1 is not verified by the trust propagation operator $\mathcal{P}_{U}$ as Table 2 proves. It is noticed, though, that both trust propagation operators coincide when both boundary trust function inputs have 
Table 1: $\mathcal{P}_{k c}$ boundary values

\begin{tabular}{c|cccc}
\hline & $(0,0)$ & $(0,1)$ & $(1,0)$ & $(1,1)$ \\
\hline$(0,0)$ & $(0,0)$ & $(0,0)$ & $(0,0)$ & $(0,0)$ \\
$(0,1)$ & $(0,0)$ & $(0,1)$ & $(0,1)$ & $(0,0)$ \\
$(1,0)$ & $(0,0)$ & $(0,1)$ & $(1,0)$ & $(0,0)$ \\
$(1,1)$ & $(0,0)$ & $(0,0)$ & $(0,0)$ & $(0,0)$ \\
\hline
\end{tabular}

Table 2: $\mathcal{P}_{U}$ boundary values

\begin{tabular}{c|cccc}
\multicolumn{1}{|c}{ Table 2: } & $\mathcal{P}_{U}$ boundary values \\
\hline & $(0,0)$ & $(0,1)$ & $(1,0)$ & $(1,1)$ \\
\hline$(0,0)$ & $(0,0)$ & $(0,1)$ & $(0,0)$ & $(0,0)$ \\
$(0,1)$ & $(0,1)$ & $(0,1)$ & $(0,1)$ & $(0,1)$ \\
$(1,0)$ & $(0,0)$ & $(0,1)$ & $(1,0)$ & $(1,1)$ \\
$(1,1)$ & $(0,0)$ & $(0,1)$ & $(1,0)$ & $(1,1)$ \\
\hline
\end{tabular}

full knowledge coverage. The reason for this resides in that the knowledge coverage-based trust propagation operator does not penalise trust information that is perfect information (see Property 2 below), while the opposite happens if the information is not perfect and the results obtained with $\mathcal{P}_{U}$ differ from those obtained with $\mathcal{P}_{U}$. Also, from Table 2 , it is obvious that $\mathcal{P}_{U}((1,0), x)=\mathcal{P}_{U}((1,1), x)$, which evidences that this uninorm trust propagation operator cannot reflect the difference of distrust degrees of the first input trust function in this cases, which is not an issue for the knowledge coverage-based trust propagation operator. Therefore, the boundary values comparative shows that the proposed trust propagation operator $\mathcal{P}_{k c}$ effectively penalises knowledge deficit, while trust information with high knowledge coverage can be retained by the knowledge coverage-based trust propagation operator, which makes the propagation result more reasonable.

Property 2 (Generalisation). If $T F_{1}=\left(t_{1}, d_{1}\right)$ and $T F_{2}=\left(t_{2}, d_{2}\right)$ are trust functions with complete knowledge coverage, i.e. $k c_{1}=k c_{2}=1$, then

$$
\mathcal{P}_{k c}\left(\left(t_{1}, d_{1}\right),\left(t_{2}, d_{2}\right)\right)=\mathcal{P}_{U}\left(\left(t_{1}, d_{1}\right),\left(t_{2}, d_{2}\right)\right)
$$

Proof.

$$
\begin{aligned}
\mathcal{P}_{k c}\left(\left(t_{1}, d_{1}\right),\left(t_{2}, d_{2}\right)\right) & =\left(U\left(\mathcal{T}\left(k c_{1}, t_{1}\right), \mathcal{T}\left(k c_{2}, t_{2}\right)\right), U\left(\mathcal{T}\left(k c_{1}, t_{1}\right), \mathcal{T}\left(k c_{2}, d_{2}\right)\right)\right) \\
& =\left(U\left(\mathcal{T}\left(1, t_{1}\right), \mathcal{T}\left(1, t_{2}\right)\right), U\left(\mathcal{T}\left(1, t_{1}\right), \mathcal{T}\left(1, d_{2}\right)\right)\right) \\
& =\left(U\left(t_{1}, t_{2}\right), U\left(t_{1}, d_{2}\right)\right) \\
& =\mathcal{P}_{U}\left(\left(t_{1}, d_{1}\right),\left(t_{2}, d_{2}\right)\right)
\end{aligned}
$$

Property 3 (Knowledge coverage absorption). When one of the input trust functions of the knowledge coverage-based trust propagation operator has no knowledge coverage then the result of the trust propagation operator is the trust function with no knowledge coverage $(0,0)$.

$$
\mathcal{P}_{k c}((1,1),(t, d))=\mathcal{P}_{k c}((t, d),(1,1))=\mathcal{P}_{k c}((0,0),(t, d))=\mathcal{P}_{k c}((t, d),(0,0))=(0,0) \forall(t, d) \in[0,1]^{2}
$$

Proof. Obvious. 
The trust propagation model can be represented as a directed weighted graph since the trust relationship is asymmetric [37]; however, the order in which the trust propagation is computed in a given indirect path involving three or more TTPs will affect the final trust estimation between nodes not directly connected in the graph. Thus, a rule needs to be set prior to such computation in practice. This study applies the sequential left-to-right direction of the indirect path connecting nodes not directly connected in the graph as show as Fig.3.

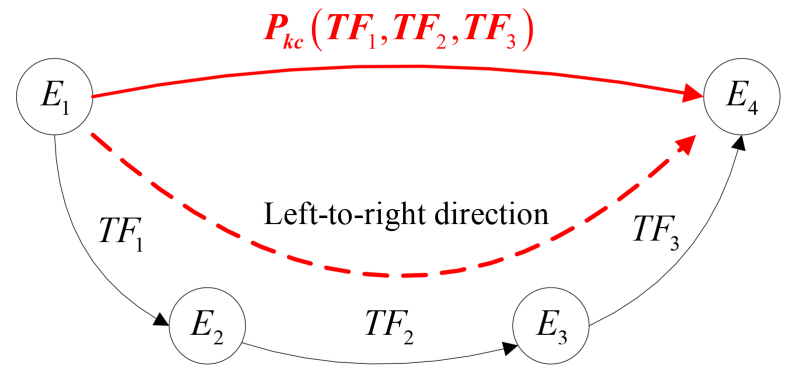

Figure 3: Knowledge coverage-based trust propagation of left-to-right direction

Comparative example 1. Let $T F_{1}=(0.1,0), T F_{2}=(0.6,0.9), T F_{3}=(0.5,0.9)$, and $T F_{4}=$ $(0.5,0.8)$ be four trust functions. Utilising the knowledge coverage-based trust propagation operator $\mathcal{P}_{k c}$, the following propagation results are obtained:

$\mathcal{P}_{k c 12}=\mathcal{P}_{k c}\left(T F_{1}, T F_{2}\right)=(0.273,0.500) ;$

$\mathcal{P}_{k c 23}=\mathcal{P}_{k c}\left(T F_{2}, T F_{3}\right)=(0.545,0.931) ;$

$\mathcal{P}_{k c 34}=\mathcal{P}_{k c}\left(T F_{3}, T F_{4}\right)=(0.500,0.800)$.

Based on Definition 8, the following ranking order isobtained: $\mathcal{P}_{k c 34}<\mathcal{P}_{k c 23}<\mathcal{P}_{k c 12}$.

The results using the uninorm trust propagation operator $\mathcal{P}_{U}$ are:

$\mathcal{P}_{U 12}=\mathcal{P}_{U}\left(T F_{1}, T F_{2}\right)=(0.005,0.008) ;$

$\mathcal{P}_{U 23}=\mathcal{P}_{U}\left(T F_{2}, T F_{3}\right)=(0.146,0.335) ;$

$\mathcal{P}_{U 34}=\mathcal{P}_{U}\left(T F_{3}, T F_{4}\right)=(0.070,0.119)$.

In this case it is $\mathcal{P}_{U 34}<\mathcal{P}_{U 12}<\mathcal{P}_{U 23}$ (based on Definition 8).

The ranking ordering of the propagated trust functions obtained with $\mathcal{P}_{k c}$ and $\mathcal{P}_{U}$ are different. Yang and Chiclana [38] proved that different order relations imply the inconsistency of different methods. Clearly, the change of distance will lead to inconsistency ranking with previous used distance. If knowledge coverage is considered a derivative of trust and distrust degrees, then there would be no reason why the propagation operator should be calculated using $(t, d)$ rather than $(t, k c)$. Hence, the present inconsistency validates our idea that the knowledge coverage is significant. 


\subsection{Knowledge coverage-based trust propagation model}

The existing propagation models rely on one single indirect linking path between unconnected nodes. In fact, the shortest indirect paths is used to assure that trust decay in the propagation is minimised [1, 20]. However, some important trust information may be lost in discarding the other longer indirect linking paths if present. In addition, the shortest length indirect path is assumed to be unique. If this is not the case, then the random choice of one of the shortest indirect paths is not persuasive. The alternative approach is to make use of all existent indirect paths, which implies that trust decay is also taken into consideration. This is the approach taken in this study to develop a multi-path weighted arithmetic averaging propagation model.

Definition 10 (Knowledge coverage-based multi-paths trust propagation model). Lets assume that $\left\{\mathcal{P}_{1}, \mathcal{P}_{2}, \ldots \mathcal{P}_{n}\right\}$ is the set of trust relationship, obtained with via the knowledge coveragebased trust propagation operator (7), associated to the set of $n$ indirect linking paths between two unconnected experts. The final trust relationship between the experts is given by

$\overline{\mathcal{P}}=\sum_{i=1}^{n} \gamma_{i} \mathcal{P}_{i}$

where

$$
\gamma_{i}=\frac{1}{k_{i} \cdot \sum_{j=1}^{n}\left(\frac{1}{k_{j}}\right)}
$$

is the weight value used to penalise the trust decay $\mathcal{P}_{i}$ associated to the length $k_{i}$ of the indirect path $i \in\{1, \ldots, n\}$.

Example 1. A company wants to choose the most appropriate cloud service supplier. To make the decision result more reasonable, they asked a group of five experts $\left\{E_{1}, E_{2}, E_{3}, E_{4}, E_{5}\right\}$ for their opinion. The group of experts is assumed to have the incomplete trust sociometric $T_{R}$ of Fig 1, with trust functions given below:

$$
T_{R}=\left[\begin{array}{cccccc}
E_{1} & E_{2} & E_{3} & E_{4} & E_{5} \\
E_{1}- & - & (0.6,0.1) & - & (0.8,0.1) \\
E_{2}(0.8,0.5) & - & - & (0.7,0.5) & - \\
E_{3}- & (0.7,0.2) & - & (0.5,0.2) & - \\
E_{4}(0.8,0.3) & - & - & - & (0.6,0.3) \\
E_{5} & - & (0.7,0.2) & (0.3,0.1) & - & -
\end{array}\right] .
$$

To obtain the complete trust network, It is necessary to get all possible indirect paths between unconnected experts and their respective lengths:

- Paths from $E_{1}$ to $E_{2}: L_{12}^{1}: E_{1} \rightarrow E_{3} \rightarrow E_{2} ; L_{12}^{2}: E_{1} \rightarrow E_{5} \rightarrow E_{2} ; L_{12}^{3}: E_{1} \rightarrow E_{5} \rightarrow E_{3} \rightarrow E_{2}$; $L_{12}^{4}: E_{1} \rightarrow E_{3} \rightarrow E_{4} \rightarrow E_{5} \rightarrow E_{2}$. 
- Paths from $E_{1}$ to $E_{4}: L_{14}^{1}: E_{1} \rightarrow E_{3} \rightarrow E_{4} ; L_{14}^{2}: E_{1} \rightarrow E_{3} \rightarrow E_{2} \rightarrow E_{4} ; L_{14}^{3}: E_{1} \rightarrow E_{5} \rightarrow$ $E_{3} \rightarrow E_{4} ; L_{14}^{4}: E_{1} \rightarrow E_{5} \rightarrow E_{2} \rightarrow E_{4} ; L_{14}^{5}: E_{1} \rightarrow E_{5} \rightarrow E_{3} \rightarrow E_{2} \rightarrow E_{4}$.

- Paths from $E_{2}$ to $E_{3}: \quad L_{23}^{1}: E_{2} \rightarrow E_{1} \rightarrow E_{3} ; L_{23}^{2}: E_{2} \rightarrow E_{1} \rightarrow E_{5} \rightarrow E_{3} ; L_{23}^{3}: E_{2} \rightarrow E_{4} \rightarrow$ $E_{1} \rightarrow E_{3} ; L_{23}^{4}: E_{2} \rightarrow E_{4} \rightarrow E_{5} \rightarrow E_{3} ; L_{23}^{5}: E_{2} \rightarrow E_{4} \rightarrow E_{1} \rightarrow E_{5} \rightarrow E_{3}$.

- Paths from $E_{2}$ to $E_{5}: L_{25}^{1}: E_{2} \rightarrow E_{1} \rightarrow E_{5} ; L_{25}^{2}: E_{2} \rightarrow E_{4} \rightarrow E_{5} ; L_{25}^{3}: E_{2} \rightarrow E_{4} \rightarrow E_{1} \rightarrow E_{5}$; $L_{25}^{4}: E_{2} \rightarrow E_{1} \rightarrow E_{3} \rightarrow E_{4} \rightarrow E_{5}$.

- Paths from $E_{3}$ to $E_{1}: L_{31}^{1}: E_{3} \rightarrow E_{2} \rightarrow E_{1} ; L_{31}^{2}: E_{3} \rightarrow E_{4} \rightarrow E_{1} ; L_{31}^{3}: E_{3} \rightarrow E_{2} \rightarrow E_{4} \rightarrow E_{1}$; $L_{31}^{4}: E_{3} \rightarrow E_{4} \rightarrow E_{5} \rightarrow E_{2} \rightarrow E_{1}$.

- Paths from $E_{3}$ to $E_{5}: L_{35}^{1}: E_{3} \rightarrow E_{4} \rightarrow E_{5} ; L_{35}^{2}: E_{3} \rightarrow E_{2} \rightarrow E_{4} \rightarrow E_{5} ; L_{35}^{3}: E_{3} \rightarrow E_{2} \rightarrow$ $E_{1} \rightarrow E_{5} ; L_{35}^{4}: E_{3} \rightarrow E_{4} \rightarrow E_{1} \rightarrow E_{5} ; L_{35}^{5}: E_{3} \rightarrow E_{2} \rightarrow E_{4} \rightarrow E_{1} \rightarrow E_{5}$.

- Paths from $E_{4}$ to $E_{2}: L_{42}^{1}: E_{4} \rightarrow E_{5} \rightarrow E_{2} ; L_{42}^{2}: E_{4} \rightarrow E_{5} \rightarrow E_{3} \rightarrow E_{2} ; L_{42}^{3}: E_{4} \rightarrow E_{1} \rightarrow$ $E_{3} \rightarrow E_{2} ; L_{42}^{4}: E_{4} \rightarrow E_{1} \rightarrow E_{5} \rightarrow E_{2} ; L_{42}^{5}: E_{4} \rightarrow E_{1} \rightarrow E_{5} \rightarrow E_{3} \rightarrow E_{2}$.

- Paths from $E_{4}$ to $E_{3}: L_{43}^{1}: E_{4} \rightarrow E_{5} \rightarrow E_{3} ; L_{43}^{2}: E_{4} \rightarrow E_{1} \rightarrow E_{3} ; L_{43}^{3}: E_{4} \rightarrow E_{1} \rightarrow E_{5} \rightarrow E_{3}$; $L_{43}^{4}: E_{4} \rightarrow E_{5} \rightarrow E_{2} \rightarrow E_{1} \rightarrow E_{3}$.

- Paths from $E_{5}$ to $E_{1}: L_{51}^{1}: E_{5} \rightarrow E_{2} \rightarrow E_{1} ; L_{51}^{2}: E_{5} \rightarrow E_{2} \rightarrow E_{4} \rightarrow E_{1} ; L_{51}^{3}: E_{5} \rightarrow E_{3} \rightarrow$ $E_{2} \rightarrow E_{1} ; L_{51}^{4}: E_{5} \rightarrow E_{3} \rightarrow E_{4} \rightarrow E_{1} ; L_{51}^{5}: E_{5} \rightarrow E_{3} \rightarrow E_{2} \rightarrow E_{4} \rightarrow E_{1}$.

- Paths from $E_{5}$ to $E_{4}: L_{54}^{1}: E_{5} \rightarrow E_{2} \rightarrow E_{4} ; L_{54}^{2}: E_{5} \rightarrow E_{3} \rightarrow E_{4} ; L_{54}^{3}: E_{5} \rightarrow E_{3} \rightarrow E_{2} \rightarrow E_{4}$; $L_{54}^{4}: E_{5} \rightarrow E_{2} \rightarrow E_{1} \rightarrow E_{3} \rightarrow E_{4}$.

To illustrate the multi-path weighted arithmetic averaging propagation model, the propagation from $E_{1}$ to $E_{2}$ is elaborated using the knowledge coverage-based trust propagation operator (8):

$$
\begin{aligned}
\mathcal{P}_{L_{12}^{1}}((0.6,0.1),(0.7,0.2)) & =(0.55,0.14) ; \\
\mathcal{P}_{L_{12}^{2}}((0.8,0.1),(0.8,0.5)) & =(0.81,0.36) ; \\
\mathcal{P}_{L_{12}^{3}}((0.8,0.1),(0.3,0.1)(0.7,0.2)) & =(0.15,0.02) ; \\
\mathcal{P}_{L_{12}^{4}}((0.6,0.1),(0.5,0.2)(0.6,0.3)(0.8,0.5)) & =(0.04,0.01) .
\end{aligned}
$$

From (9), the final trust relationship of $E_{1}$ to $E_{2}$ is:

$$
\overline{\mathcal{P}}_{12}=(0.46,0.16)
$$

Similarly, all missing trust degree in the incomplete trust sociometric $T_{R}$ are computed, resulting in 
the following complete trust sociometric $T_{C}$ as:

$$
T_{C}=\left[\begin{array}{ccccc}
E_{1} & E_{2} & E_{3} & E_{4} & E_{5} \\
E_{1}- & (0.46,0.16) & (0.60,0.10) & (0.32,0.20) & (0.80,0.10) \\
E_{2}(0.80,0.50) & - & (0.38,0.10) & (0.70,0.50) & (0.62,0.18) \\
E_{3}(0.58,0.28) & (0.70,0.20) & - & (0.50,0.20) & (0.62,0.17) \\
E_{4}(0.80,0.30) & (0.53,0.20) & (0.41,0.11) & - & (0.60,0.30) \\
E_{5}(0.35,0.20) & (0.70,0.20) & (0.30,0.10) & (0.28,0.19) & -
\end{array}\right] .
$$

\section{A trust order induced consensus reaching mechanism for group decision making}

After constructing the completed trust network of experts, a group decision including a group consensus process becomes possible. Herrera-Viedma et al. [5] proposed a three-dimensional soft consensus approach for measures consensus, which is widely applied in consensus reaching processes. Once the consensus degrees of all experts have reached the minimum acceptable consensus threshold for the group, the selection process would be activated. In most situations, some experts do not reach the minimum acceptable consensus threshold at first. Therefore, inconsistent opinions detection and recommendation mechanism have been developed to generate advice to help the inconsistent experts increase their consensus. Existing research about recommendation mechanisms apply the average acceptance method, which is based on the unreasonable forced acceptance by the inconsistent expert of the recommendation they receive [15]. It is unreasonable because the inconsistent experts may have strong feelings about loosing their independence by changing their opinions for the solely benefit of reaching consensus without any other justification to support the validity of such recommendations. The construction of a trust relationship network provides support for addressing this issue because, in practical situations, experts acceptance of advice has a positive correlation with the trust degree of the experts from whom the advice comes from.

Inspired by this, a subjective and objective trust ordered recommendation mechanism is proposed to generate advice based not only on an objective weight vector, obtained from the consensus degree of experts' decision matrices, but also on a subjective weight vector, obtained from the trust relationship between experts. The decision-making processes based on the subject and objective trust ordered recommendation mechanism can be show as Fig.4.

\subsection{The consensus degree measurement method with trust functions}

Consensus degree of expert $e^{h}$ on elements level. The pairwise consensus levels of experts $e^{h}$ and $e^{k}$ on the alternative $x_{i}$ and attribute $c_{j}$ is computed as:

$$
C E_{i j}\left(e^{h}, e^{k}\right)=1-\frac{\left(\left|t_{i j}^{h}-t_{i j}^{k}\right|+\left|d_{i j}^{h}-d_{i j}^{k}\right|\right)}{2}
$$




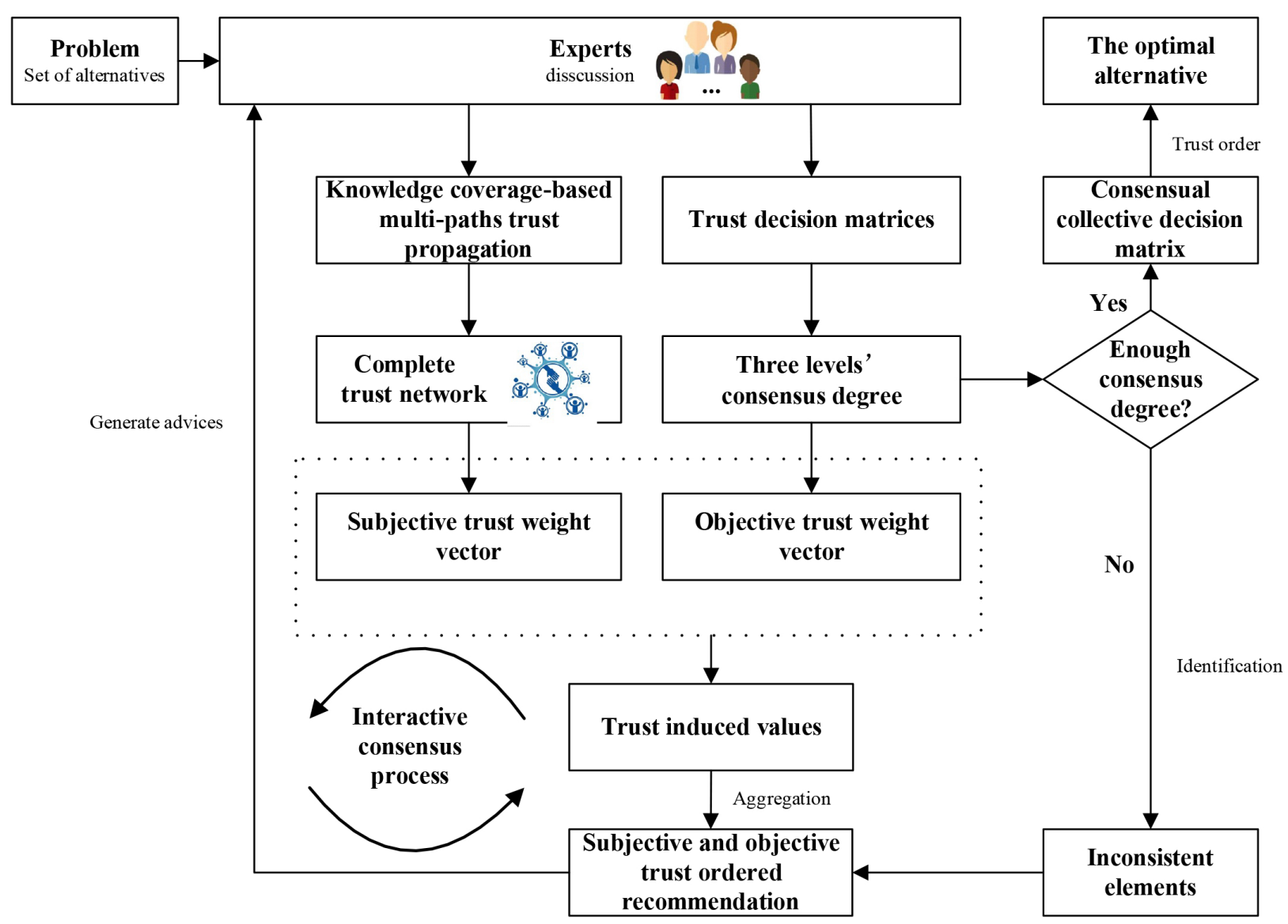

Figure 4: A trust order induced recommendation mechanism for SN-GDM

The consensus degree of expert $E^{h}$ with the decision group on the alternative $x_{i}$ and attribute $c_{j}$ is:

$$
A C E_{i j}^{h}=\frac{1}{l-1} \sum_{h \neq k, k=1}^{l} C E_{i j}\left(e^{h}, e^{k}\right)
$$

Consensus degree of expert $e^{h}$ on alternatives level. The pairwise consensus levels of experts $e^{h}$ and $e^{k}$ on the alternative $x_{i}$ is computed as:

$C A_{i}\left(e^{h}, e^{k}\right)=\frac{1}{n} \sum_{j=1}^{n}\left(1-\frac{\left|t_{i j}^{h}-t_{i j}^{k}\right|+\left|d_{i j}^{h}-d_{i j}^{k}\right|}{2}\right)$

The consensus degree of expert $E^{h}$ with the decision group on the alternative $x_{i}$ is:

$$
A C A_{i}^{h}=\frac{1}{l-1} \sum_{h \neq k, k=1}^{l} C A_{i}\left(e^{h}, e^{k}\right)
$$

Consensus degree of expert $e^{h}$ on decision matrix. The pairwise consensus levels of experts $e^{h}$ and $e^{k}$ on the decision matrix is computed as:

$$
C D\left(e^{h}, e^{k}\right)=\frac{1}{m \cdot n} \sum_{i=1}^{m} \sum_{j=1}^{n}\left(1-\frac{\left|t_{i j}^{h}-t_{i j}^{k}\right|+\left|d_{i j}^{h}-d_{i j}^{k}\right|}{2}\right)
$$


The bigger this pairwise consensus degree is, the more trust there is between these two experts.

The consensus degree of expert $E^{h}$ with the decision group on the decision matrix is:

$A C D^{h}=\frac{1}{l-1} \sum_{h \neq k, k=1}^{l} C D\left(e^{h}, e^{k}\right)$

The bigger the $A C D^{h}$ is, the higher the consensus of the expert is.

Practically, it is rare to reach perfect consensus $(\vartheta=1)$ in group decision making situations. Meanwhile, group consensus usually requires 'most of'/'as many as possible' experts to reach consensus. Therefore, the minimum consensus threshold value should verify $\vartheta \in[0.5,1)$. Once $A C D^{h} \geq \vartheta \forall h$, the consensus interactive process ends and the selection process is stimulated.

Example 2. (Example 1 continuation) The cloud service selection involves four alternatives, $A_{i}=\left\{A_{1}, A_{2}, A_{3}, A_{4}\right\}$, and four criteria, $c_{i}=\left\{c_{1}, c_{2}, c_{3}, c_{4}\right\}$, are employed: security, cost, usability and performance, with associated weight vector $w=(0.36,0.24,0.18,0.22)^{T}$. WE are assuming the following five decision matrixes of experts:

$$
\begin{aligned}
& R^{1}=\left[\begin{array}{llll}
(0.3,0.5) & (0.2,0.7) & (0.5,0.4) & (0.2,0.3) \\
(0.6,0.6) & (0.3,0.4) & (0.5,0.5) & (0.3,0.6) \\
(0.2,0.7) & (0.5,0.6) & (0.6,0.5) & (0.2,0.6) \\
(0.5,0.8) & (0.4,0.4) & (0.6,0.6) & (0.4,0.8)
\end{array}\right] \\
& R^{2}=\left[\begin{array}{cccc}
(0.3,0.4) & (0.2,0.7) & (0.7,0.4) & (0.5,0.6) \\
(0.4,0.6) & (0.5,0.5) & (0.5,0.4) & (0.4,0.5) \\
(0.4,0.5) & (0.4,0.8) & (0.2,0.6) & (0.5,0.5) \\
(0.6,0.5) & (0.5,0.3) & (0.3,0.8) & (0.6,0.7)
\end{array}\right] \\
& R^{3}=\left[\begin{array}{llll}
(0.3,0.6) & (0.3,0.8) & (0.5,0.6) & (0.3,0.7) \\
(0.5,0.6) & (0.6,0.3) & (0.4,0.4) & (0.5,0.6) \\
(0.3,0.4) & (0.6,0.7) & (0.3,0.6) & (0.4,0.5) \\
(0.3,0.6) & (0.4,0.5) & (0.5,0.4) & (0.5,0.6)
\end{array}\right] \\
& R^{4}=\left[\begin{array}{llll}
(0.5,0.4) & (0.4,0.5) & (0.6,0.5) & (0.4,0.5) \\
(0.3,0.5) & (0.2,0.4) & (0.6,0.5) & (0.5,0.4) \\
(0.6,0.7) & (0.4,0.7) & (0.4,0.7) & (0.3,0.7) \\
(0.5,0.7) & (0.4,0.6) & (0.4,0.8) & (0.5,0.6)
\end{array}\right] \\
& R^{5}=\left[\begin{array}{llll}
(0.6,0.3) & (0.5,0.5) & (0.7,0.6) & (0.6,0.5) \\
(0.7,0.2) & (0.6,0.2) & (0.3,0.9) & (0.5,0.4) \\
(0.6,0.6) & (0.7,0.8) & (0.9,0.7) & (0.8,0.5) \\
(0.8,0.5) & (0.6,0.8) & (0.2,0.8) & (0.7,0.5)
\end{array}\right]
\end{aligned}
$$

The following consensus values are obtained: 
Consensus degree of expert $e^{h}$ on elements level.

$$
\begin{aligned}
& A C E_{i j}^{1}=\left[\begin{array}{cccc}
0.875 & 0.863 & 0.875 & 0.738 \\
0.850 & 0.838 & 0.875 & 0.850 \\
0.788 & 0.863 & 0.775 & 0.800 \\
0.813 & 0.863 & 0.775 & 0.813
\end{array}\right] \\
& A C E_{i j}^{2}=\left[\begin{array}{cccc}
0.888 & 0.863 & 0.875 & 0.838 \\
0.850 & 0.825 & 0.863 & 0.900 \\
0.838 & 0.875 & 0.788 & 0.850 \\
0.838 & 0.813 & 0.838 & 0.875
\end{array}\right] \\
& A C E_{i j}^{3}=\left[\begin{array}{cccc}
0.838 & 0.838 & 0.875 & 0.800 \\
0.863 & 0.838 & 0.850 & 0.900 \\
0.788 & 0.888 & 0.825 & 0.863 \\
0.788 & 0.875 & 0.738 & 0.900
\end{array}\right] \\
& A C E_{i j}^{4}=\left[\begin{array}{cccc}
0.863 & 0.838 & 0.900 & 0.863 \\
0.800 & 0.800 & 0.838 & 0.900 \\
0.813 & 0.888 & 0.825 & 0.800 \\
0.850 & 0.863 & 0.850 & 0.900
\end{array}\right] \\
& A C E_{i j}^{5}=\left[\begin{array}{cccc}
0.788 & 0.800 & 0.875 & 0.813 \\
0.688 & 0.800 & 0.675 & 0.900 \\
0.825 & 0.838 & 0.688 & 0.738 \\
0.763 & 0.738 & 0.800 & 0.813
\end{array}\right]
\end{aligned}
$$

Consensus degree of expert $e^{h}$ on alternatives level.

$$
\begin{aligned}
& A C A_{i}^{1}=(0.838,0.853,0.806,0.816)^{T} ; \\
& A C A_{i}^{2}=(0.866,0.859,0.838,0.841)^{T} ; \\
& A C A_{i}^{3}=(0.838,0.863,0.841,0.825)^{T} ; \\
& A C A_{i}^{4}=(0.866,0.834,0.831,0.866)^{T} ; \\
& A C A_{i}^{5}=(0.819,0.766,0.772,0.778)^{T} .
\end{aligned}
$$

Consensus degree of expert $e^{h}$ on decision matrix.

$$
A C D^{1}=0.828 ; A C D^{2}=0.851 ; A C D^{3}=0.841 ; A C D^{4}=0.849 ; A C D^{5}=0.784 .
$$

Assuming the minimum consensus degree for the company of $\vartheta=0.8$, then expert $e^{5}$ will be asked to revise his/her inconsistent trust decision opinions. 


\subsection{Subjective and objective trust ordered recommendation mechanism}

The subjective and objective trust ordered recommendation mechanism involves three steps: (1) Identifying the decision opinions to be revised; (2) Obtaining the subjective and objective trust relationship of experts; and (3) Generating the subjective and objective trust order based recommended advice.

\subsubsection{Identifying the decision opinions need to be revised}

For identifying the decision opinions to be revised, a three-level identification method was introduced to locate the trust decision opinions that contribute less to group consensus [5].

Level 1. Identify experts with consensus degrees below the minimum threshold value $\vartheta$ :

$$
E X P C H=\left\{h \mid A C D^{h}<\vartheta\right\}
$$

Let $p=l-\# E X P C H$ be the number of experts with consensus degrees not below the minimum threshold value $\vartheta$. For convenience, this set of experts will be denoted as $\left\{e^{t} \mid t=1, \ldots, e^{p}\right\}$, and will refer to a the set of consistent experts.

Level 2. For experts in $E X P C H$, their alternatives with consensus below $\vartheta$ are identified:

$$
A L T=\left\{(h, i) \mid h \in E X P C H \wedge A C A_{i}^{h}<\vartheta\right\} .
$$

Level 3. Finally, the set of trust decision opinions to be revised is:

$$
A P S=\left\{(h, i, j) \mid(h, i) \in A L T \wedge A C E_{i j}^{h}<\vartheta\right\} .
$$

Example 3. (Example 2 continuation) The set of trust decision opinions to be revised is $A P S=$ $\{(5,2,1),(5,2,3),(5,3,3),(5,3,4),(5,4,1),(5,4,2)\}$

\subsubsection{Obtaining the subjective and objective trust relationship of experts}

Objective trust relationship refers to the trust relationship derived from the experts's decision opinions, while subjective trust relationship refers to the trust relationship derived derived from the completed trust sociometric relationship between the experts. Both subjective and objective trust relationship of experts need to be obtained for generating advice.

Definition 11 (Objective trust degree (OTD)). The pairwise objective trust degree between experts $e^{h}$ and $e^{k}$ is their consensus degree on the decision matrix level:

$O T D^{h k}=C D\left(e^{h}, e^{k}\right)$ 
Expert $e^{h}(h \in E X P C H)$ is associated the following objective trust weight value towards the consistent expert $e^{t}$ :

$\mu_{h}^{t}=\frac{O T D^{h t}}{\sum_{t=1}^{p} O T D^{h t}}$.

If the trust sociometric matrix $T_{C}$ between experts is incomplete, then the proposed knowledge coverage-based multi-paths trust propagation model is applied to complete it.

Definition 12 (Subjective trust degree (STD)). The pairwise subjective trust degree between experts $e^{h}$ and $e^{k}$ is

$S T D^{h k}=T_{C}\left(e^{h}, e^{k}\right)$

As per (6), the positive trust score of $S T D^{h k}$ is denoted by $P T S^{h k}$. Then expert $e^{h}(h \in E X P C H)$ can be associated the following subjective trust weight value towards consistent expert $e^{t}$ :

$\nu_{h}^{t}=\frac{P T S^{h t}}{\sum_{t=1}^{p} P T S^{h t}}$.

Based on the objective and subjective trust weight vectors, this study proposes the following definition of collective trust induced values.

Definition 13 (The collective trust induced values (CT)). The collective trust induced value of inconsistent expert $e^{h}$ with respect to the consistent expert $e^{t}$ is:

$C T_{h}^{t}=(1-\varphi) \cdot \mu_{h}^{t}+\varphi \cdot \nu_{h}^{t}, \forall t=1,2, \ldots, p$

where $\varphi \in[0,1]$ is a parameter that controls subjective and objective degrees.

Example 4. (Example 3 continuation) As per (14), we have:

$$
\text { OTD }=\left[\begin{array}{ccccc}
- & 0.853 & 0.866 & 0.859 & 0.734 \\
0.853 & - & 0.875 & 0.869 & 0.806 \\
0.866 & 0.875 & - & 0.850 & 0.775 \\
0.859 & 0.869 & 0.850 & - & 0.819 \\
0.734 & 0.806 & 0.775 & 0.819 & -
\end{array}\right]
$$

The objective trust weight vector of expert $e^{5}$ towards the other consistent experts is:

$$
\mu_{5}^{t}=(0.234,0.257,0.247,0.261) .
$$

As per Example 1, the subjective trust weight vector of expert $e^{5}$ towards the other consistent experts is:

$$
\nu_{5}^{t}=(0.241,0.267,0.263,0.230)
$$


With $\varphi=0.5$, the trust induced values of expert $e^{5}$ towards the other consistent experts is:

$$
C T_{5}^{t}=(0.238,0.262,0.255,0.245)
$$

\subsubsection{Generating the subjective and objective trust order based recommended advice}

Existing advice generating methods over-prioritised the objective trust relations, i.e. the trust relation obtained from the consensus level of the decision opinions. However, expert may have a higher subjective trust degree towards other experts from previous personal experiences. For modelling this behaviour, a subjective and objective trust order-based advice generating method is proposed based on the induced ordered weighted averaging (IOWA) operator proposed by Yager and Filev [39].

For any $(h, i, j) \in A S P$, inconsistent expert $e^{h}$ will be provided with the following trust ordered based personalised advice:

"According to your trust relationship, you are recommended to change your evaluation for alternative $x_{i}$ under attribute $c_{j}, r_{i j}^{h}=\left(t_{i j}^{h}, d_{i j}^{h}\right)$, to the value $r r_{i j}^{h}=\left(r t_{i j}^{h}, r d_{i j}^{h}\right), "$

where

$r r_{i j}^{h}=\left(r t_{i j}^{h}, r d_{i j}^{h}\right)=\left((1-\delta) \cdot t_{i j}^{h}+\delta t_{i j}^{\Delta},(1-\delta) \cdot d_{i j}^{h}+\delta d_{i j}^{\Delta}\right)$

where $\delta \in[0,1]$ is a parameter to control the personalised advice degree, and $\left(t_{i j}^{\Delta}, d_{i j}^{\Delta}\right)$ are computed via a coverage-trust IOWA (CT-IOWA) operator:

Definition 14. The CT-IOWA aggregated result from a set a set $\left\{T F_{i j}^{t} \mid T F_{i j}^{t}=\left(t_{i j}^{t}, d_{i j}^{t}\right)\right\}(t=1, \ldots, p)$ of decision opinions is

$C T-I O W A_{w_{t}}\left(\left\langle C T_{h}^{1}, T F_{i j}^{1}\right\rangle, \ldots,\left\langle C T_{h}^{p}, T F_{i j}^{p}\right\rangle\right)=\left(\sum_{t=1}^{p} \xi_{t} t_{i j}^{\sigma(t)}, \sum_{t=1}^{p} \xi_{t} d_{i j}^{\sigma(t)}\right)$

with $\sigma_{(t)}$ is the permutation of $(t=1, \ldots, p)$ such that $C T_{h}^{\sigma_{(t)}} \geq C T_{h}^{\sigma(t+1)}$, and

$\xi_{t}=Q\left(\frac{T(t)}{T(p)}\right)-Q\left(\frac{T(t-1)}{T(p)}\right)$

where $Q$ is the membership function of a linguistic quantifier used to represent a soft majority concept such as 'most of' with $Q(r)=r^{2}$, and $T(t)=C T_{h}^{\sigma_{(1)}}+\ldots+C T_{h}^{\sigma_{(t)}}(t=1, \ldots, p)$.

Example 5. (Example 4 continuation) With the parameter value $\delta=0.5$, the subjective and objective trust order based recommended advice for expert $e^{5}$ are:

According to your trust relationship, you are recommended to change your evaluation for

- alternative $x_{2}$ under attribute $c_{1}$ to the value $(0.566,0.392)$ 
- alternative $x_{2}$ under attribute $c_{3}$ to the value $(0.397,0.665)$

- alternative $x_{3}$ under attribute $c_{3}$ to the value $(0.603,0.651)$

- alternative $x_{3}$ under attribute $c_{4}$ to the value $(0.604,0.523)$

- alternative $x_{4}$ under attribute $c_{1}$ to the value $(0.654,0.546)$

- alternative $x_{4}$ under attribute $c_{2}$ to the value $(0.525,0.601)$

\subsubsection{Second round for reaching consensus}

Once the personalised recommendations have been generated and accepted, the second-round process of trust ordered consensus interaction mechanism will be adopted to check whether the group decision opinion of all experts reaches consensus. If it reaches the group consensus, then the selection process will be triggered; otherwise, a further consensus interactive mechanism is needed.

Example 6. (Example 5 continuation) After accepting the trust based recommendations, the new experts consensus degrees are (using the same $\varphi_{i}=0.5$ ):

$$
A C D^{1^{\prime}}=0.841 ; A C D^{2^{\prime}}=0.863 ; A C D^{3^{\prime}}=0.854 ; A C D^{4^{\prime}}=0.862 ; A C D^{5^{\prime}}=0.834 .
$$

The below shows that the final average consensus degree $A C D^{5^{\prime}}$ calculated with different subjective and objective parameters $\varphi_{i}$ will increase. Indeed, the final average consensus degree $A C D^{5^{\prime}}$ with different parameter values $\varphi_{i}=\{0,0.2,0.4,0.6,0.8,1,0.5\}$ are provided in Table 3 , which shows that in all cases is higher than before the feedback process.

\begin{tabular}{l|lllllll}
\multicolumn{7}{c}{ Table 3: $A C D^{5^{\prime}}$ of different parameters $\varphi_{i}$} \\
\hline$\varphi_{i}$ & 0 & 0.2 & 0.4 & 0.6 & 0.8 & 1 & 0.5 \\
\hline$A C D^{5^{\prime}}$ & 0.8328 & 0.8335 & 0.8337 & 0.8337 & 08337 & 08337 & 08337 \\
\hline
\end{tabular}

\section{Selection process}

One the group consensus has been reached, the selection process is triggered. The individual decision opinions are aggregated with a weighted arithmetic averaging (WAA), whose weights are the final average consensus degrees of experts $\omega^{h}=A C D^{h} / \sum_{k=1}^{l} A C D^{k}(h=1, \ldots, l)$., to derive the collective decision matrix:

$\bar{r}_{i j}=\sum_{h=1}^{l} \omega^{h} r_{i j}^{h}$

If the criteria weight vector is $c_{j}(j=1, \ldots, n)$, then the alternatives final evaluations will be:

$\tilde{A}_{i}=\sum_{j=1}^{n} w_{j} \bar{r}_{i j}$ 
Finally, based on Definition 8, the ranking of alternatives is derived.

Example 7. (Example 6 continuation) Based on the final average consensus degree of experts, the collective decision matrix can be calculated as:

$\bar{r}_{i j}=\left[\begin{array}{cccc}c_{1} & c_{2} & c_{3} & c_{4} \\ A_{1}(0.360,0.438) & (0.279,0.669) & (0.625,0.473) & (0.424,0.579) \\ A_{2}(0.446,0.564) & (0.472,0.405) & (0.485,0.454) & (0.432,0.527) \\ A_{3}(0.408,0.523) & (0.481,0.743) & (0.338,0.608) & (0.428,0.543) \\ A_{4}(0.540,0.583) & (0.466,0.424) & (0.376,0.692) & (0.554,0.658)\end{array}\right]$

As the criteria weight vector is $w_{j}=(0.36,0.24,0.18,0.22)^{T}$, the final assessment of alternatives are:

$$
\tilde{A}_{1}=(0.402,0.531) ; \tilde{A}_{2}=(0.454,0.498) ; \tilde{A}_{3}=(0.417,0.596) ; \tilde{A}_{4}=(0.496,0.581) .
$$

Based Definition 8, the ranking of alternatives is: $\tilde{A}_{2} \succ \tilde{A}_{4} \succ \tilde{A}_{1} \succ \tilde{A}_{3}$.

\section{Conclusion}

A novel trust ordered recommendation mechanism for SN-GDM with multi-paths knowledge coverage-based propagation has been proposed. This SN-GDM method has the following advantages:

1) The concept of knowledge coverage and a new ranking method of trust functions have been proposed in this study. As the degree of knowledge coverage shows the credibility of trust information, the results of the proposed knowledge coverage-based trust propagation are considered reasonable.

2) Among the properties of the proposed knowledge coverage-based trust propagation operator, based on uninorm and t-norm, are: boundary conditions are more reasonable than the ones of existing trust propagation operators; it generalises the previous uninorm trust propagation operator when trust information has perfect knowledge.

3) A knowledge coverage-based multi-paths trust propagation model based on weighted arithmetic averaging (WAA) operator has been developed. Compared with the existing multi-path trust propagation model, the proposed model has the advantage of avoiding ignoring trust information due to the implementation of all trust paths and the penalisation of path's trust decay.

4) A trust order induced consensus reaching mechanism for GDM is developed in this study. In this mechanism, subjective trust degrees are from experts' completed trust sociometric, while objective trust degrees are derived from their consensus degree. A trust order induced recommendation mechanism is proposed by combining subjective and objective weights. This recommendation mechanism allows the inconsistent experts to accept the advices they trust, making it reasonable and feasible in practice. 


\section{Acknowledgements}

The authors are very grateful to the anonymous referees for their valuable comments and suggestions. This work was supported by National Natural Science Foundation of China (NSFC) under Grants No.72001134, 71971135, 71571166, 716010161.

\section{References}

[1] J. Wu, F. Chiclana, H. Fujita, E. Herrera-Viedma, A visual interaction consensus model for social network group decision making with trust propagation, Knowledge-Based Systems 122 (2017) 3950.

[2] N. H. Kamis, F. Chiclana, J. Levesley, Geo-uninorm consistency control module for preference similarity network hierarchical clustering based consensus model, Knowledge-Based Systems 162 (2018) 103-114.

[3] F. Chiclana, J. Levesley, N. Hanimah Kamis, Preference similarity network structural equivalence clustering based consensus model, Applied Soft Computing 67 (2017) 706-720.

[4] F. Herrera, E. Herrera-Viedma, et al., A model of consensus in group decision making under linguistic assessments, Fuzzy sets and Systems 78 (1) (1996) 73-87.

[5] E. Herrera-Viedma, S. Alonso, F. Chiclana, F. Herrera, A consensus model for group decision making with incomplete fuzzy preference relations, IEEE Transactions on fuzzy Systems 15 (5) (2007) 863-877.

[6] J. Wu, Y. Liu, C. Liang, A consensus-and harmony-based feedback mechanism for multiple attribute group decision making with correlated intuitionistic fuzzy sets, International Transactions in Operational Research 22 (6) (2015) 1033-1054.

[7] J. Wu, Q. Sun, H. Fujita, F. Chiclana, An attitudinal consensus degree to control the feedback mechanism in group decision making with different adjustment cost, Knowledge-Based Systems 164 (2019) 265-273.

[8] M. Cao, J. Wu, F. Chiclana, R. Ureña, E. Herrera-Viedma, A personalized consensus feedback mechanism based on maximum harmony degree, IEEE Transactions on Systems Man \& Cybernetics Systems. DOI: 10.1109/TSMC.2019.2960052.

[9] S. Alonso, E. Herrera-Viedma, F. Chiclana, F. Herrera, A web based consensus support system for group decision making problems and incomplete preferences, Information Sciences 180 (23) (2010) 4477-4495. 
[10] I. J. Pérez, F. J. Cabrerizo, S. Alonso, E. Herrera-Viedma, A new consensus model for group decision making problems with non-homogeneous experts, IEEE Transactions on Systems, Man, and Cybernetics: Systems 44 (4) (2014) 494-498.

[11] J. Wu, M. Cao, F. Chiclana, Y. Dong, E. Herrera-Viedma, An optimal feedback model to prevent manipulation behaviours in consensus under social network group decision making, IEEE Transactions on Fuzzy Systems. DOI: 10.1109/TFUZZ.2020.2985331.

[12] A. Sallaberry, F. Zaidi, G. Melançon, Model for generating artificial social networks having community structures with small-world and scale-free properties, Social Network Analysis and Mining 3 (3) (2013) 597-609.

[13] Y. Dong, Q. Zha, H. Zhang, G. Kou, H. Fujita, F. Chiclana, E. Herrera-Viedma, Consensus reaching in social network group decision making: Research paradigms and challenges, KnowledgeBased Systems 162 (2018) 3-13.

[14] J. Wu, X. Li, F. Chiclana, R. R. Yager, An attitudinal trust recommendation mechanism to balance consensus and harmony in group decision making, IEEE Transactions on Fuzzy Systems 27 (11) (2019) 2163-2175.

[15] Y. Liu, C. Liang, F. Chiclana, J. Wu, A trust induced recommendation mechanism for reaching consensus in group decision making, Knowledge-Based Systems 119 (2017) 221-231.

[16] W. Jiang, J. Wu, F. Li, G. Wang, H. Zheng, Trust evaluation in online social networks using generalized network flow, IEEE Transactions on Computers 65 (3) (2016) 952-963.

[17] E. Gray, J.-M. Seigneur, Y. Chen, C. Jensen, Trust propagation in small worlds, in: International conference on trust management, Springer, 2003, pp. 239-254.

[18] P. Victor, Trust networks for recommender systems, Ph.D. thesis, Ghent University (2010).

[19] R. Guha, R. Kumar, P. Raghavan, A. Tomkins, Propagation of trust and distrust, in: Proceedings of the 13th international conference on World Wide Web, ACM, 2004, pp. 403-412.

[20] J. Wu, R. Xiong, F. Chiclana, Uninorm trust propagation and aggregation methods for group decision making in social network with four tuple information, Knowledge-Based Systems 96 (2016) 29-39.

[21] Y.-W. Du, N. Yang, J. Ning, Ifs/er-based large-scale multiattribute group decision-making method by considering expert knowledge structure, Knowledge-Based Systems 162 (2018) 124135. 
[22] H. Nguyen, A new knowledge-based measure for intuitionistic fuzzy sets and its application in multiple attribute group decision making, Expert Systems with Applications 42 (22) (2015) 87668774 .

[23] R. Ureña, F. Chiclana, H. Fujita, E. Herrera-Viedma, Confidence-consistency driven group decision making approach with incomplete reciprocal intuitionistic preference relations, KnowledgeBased Systems 89 (2015) 86-96.

[24] X. Liu, Y. Xu, R. Montes, F. Herrera, Social network group decision making: Managing selfconfidence-based consensus model with the dynamic importance degree of experts and trust-based feedback mechanism, Information Sciences 505 (2019) 215-232.

[25] Q. Zha, Y. Dong, H. Zhang, F. Chiclana, E. Herrera-Viedma, A personalized feedback mechanism based on bounded confidence learning to support consensus reaching in group decision making, IEEE Transactions on Systems, Man, \& Cybernetics: Systems. DOI: 10.1109/TSMC.2019.2945922

[26] Y. Dong, W. Liu, F. Chiclana, G. Kou, E. Herrera-Viedma, Are incomplete and self-confident preference relations better in multicriteria decision making? a simulation-based investigation, Information Sciences 492 (2019) 40-57.

[27] P. Victor, C. Cornelis, M. De Cock, P. D. S. Paulo, Gradual trust and distrust in recommender systems, Fuzzy Sets \& Systems 160 (10) (2009) 1367-1382.

[28] G. Wang, J. Wu, Multi-dimensional evidence-based trust management with multi-trusted paths, Future Generation Computer Systems 27 (5) (2011) 529-538.

[29] M. Mao, J. Lu, G. Zhang, J. Zhang, Multirelational social recommendations via multigraph ranking, IEEE transactions on cybernetics 47 (12) (2016) 4049-4061.

[30] P. Victor, C. Cornelis, M. D. Cock, E. Herrera-Viedma, Practical aggregation operators for gradual trust and distrust, Fuzzy Sets \& Systems 184 (1) (2011) 126-147.

[31] Y. Zhang, Z. Wu, H. Chen, H. Sheng, J. Ma, Mining target marketing groups from users' web of trust on epinions., in: AAAI Spring Symposium: Social Information Processing, 2008, p. 116.

[32] C.-W. Hang, Y. Wang, M. P. Singh, Operators for propagating trust and their evaluation in social networks, in: Proceedings of The 8th International Conference on Autonomous Agents and Multiagent Systems-Volume 2, International Foundation for Autonomous Agents and Multiagent Systems, 2009, pp. 1025-1032.

[33] M. Ghavipour, M. R. Meybodi, Trust propagation algorithm based on learning automata for inferring local trust in online social networks, Knowledge-Based Systems 143 (2018) 307-316. 
[34] J. Wu, F. Chiclana, E. Herrera-Viedma, Trust based consensus model for social network in an incomplete linguistic information context, Applied Soft Computing 35 (2015) 827-839.

[35] N. Capuano, F. Chiclana, H. Fujita, E. Herrera-Viedma, V. Loia, Fuzzy group decision making with incomplete information guided by social influence, IEEE Transactions on Fuzzy Systems 26 (3) (2018) 1704-1718.

[36] T. Gerstenkorn, J. Mańko, Correlation of intuitionistic fuzzy sets, Fuzzy sets and systems 44 (1) (1991) 39-43.

[37] A. Jøsang, C. Keser, T. Dimitrakos, Can we manage trust?, in: International Conference on Trust Management, Springer, 2005, pp. 93-107.

[38] Y. Yang, F. Chiclana, Consistency of 2d and 3d distances of intuitionistic fuzzy sets, Expert Systems with Applications 39 (10) (2012) 8665-8670.

[39] R. R. Yager, D. P. Filev, Induced ordered weighted averaging operators, IEEE Transactions on Systems, Man, and Cybernetics, Part B (Cybernetics) 29 (2) (1999) 141-150. 